\title{
Review Article \\ Nontuberculous Mycobacteria in Saudi Arabia and Gulf Countries: A Review
}

\author{
Hawra Al-Ghafli and Sahal Al-Hajoj \\ Department of Infection and Immunity, King Faisal Specialist Hospital and Research Center, Riyadh, Saudi Arabia \\ Correspondence should be addressed to Sahal Al-Hajoj; hajoj@kfshrc.edu.sa
}

Received 7 November 2016; Revised 5 January 2017; Accepted 1 February 2017; Published 27 February 2017

Academic Editor: Isabella Annesi-Maesano

Copyright (C) 2017 Hawra Al-Ghafli and Sahal Al-Hajoj. This is an open access article distributed under the Creative Commons Attribution License, which permits unrestricted use, distribution, and reproduction in any medium, provided the original work is properly cited.

\begin{abstract}
Nontuberculous Mycobacteria (NTM) are causing growing health problems worldwide. This is indicated by an increasing amount of scientific reports showing not only well-identified species reemerging but also emergence of new species. The emergence and reemergence of NTM are particularly worrying in developing countries due to scarce published data and improper identification. Here we aimed to examine the main epidemiological aspects and diagnostic challenges associated with NTM in countries of the Gulf Cooperation Council (GCC) and compare these findings to the international arena findings. Data revealed that countries of the GCC are largely dominated by rapidly growing mycobacteria species such as M. fortuitum (29\%) and M. abscessus (17\%) with high rate of definitive respiratory diseases. On the other hand, most of the developed countries are dominated by slowly growing mycobacteria such as MAC, M. kansasii, and M. gordonae. More efforts are needed, however, to gain insights into NTM issues in countries of the GCC.
\end{abstract}

\section{Introduction}

Mycobacterium is a large ancient genus of Actinobacteria; most members of Mycobacterium are described as opportunistic organisms widely spread in our planet either as water, soil, or plant inhabitants [1]. Despite the long existence of mycobacteria, the subidentification of approximately 150 Mycobacterium species had only been possible in the last two decades [2]. This is largely attributed to the emergence of new identification and fingerprinting approaches [3]. These fingerprinting approaches are time and labor efficient and provide accurate and reliable results, unlike prior descriptive bacterial taxonomy approaches.

The majority of identified mycobacterial species exhibit various levels of pathogenic impact towards humans. By far the most recognized pathogenic species in this genus is Mycobacterium tuberculosis (M. tuberculosis). M. tuberculosis is responsible for the most deadly mycobacterial infectious disease, tuberculosis (TB), with at least 1.5 million reported deaths worldwide [4]. However, the rest of the identified mycobacterial species do not cause TB and hence they are referred to as Mycobacteria Other Than Tuberculosis
(MOTT). Other names designated to MOTT include Atypical Mycobacteria (ATM) and Nontuberculous Mycobacteria (NTM)

Most NTM species are aerobic, nonmotile organisms, with rigid and thick cell walls [5]. The thickness of NTM cell wall functions as a natural protective shield against disinfectants and antibiotics $[5,6]$. These characteristics enable them to thrive in most natural resources surrounding humans. NTM can be isolated from domestic and animal products as well as artificial built systems, including shower streams and medical devices [7]. This explains the increasing number of reported NTM infections that occur upon direct ingestion, inhalation, and incubation of mycobacterial virulent agents [8].

In the past years, a sharp increase of reported NTM infections has been noted at a global scale in both immunocompromised and immunocompetent individuals [9-16]. The issue of NTM is in particular worrying in developing regions due to scarce published data and improper identification. In addition, a clear and concise view to the main issues and trends regarding NTM in countries of the Gulf Cooperation Council (GCC) is lacking in the literature. To 
TABLE 1: Species distribution of NTM in the Gulf region.

\begin{tabular}{|c|c|c|c|c|c|c|}
\hline \multicolumn{7}{|c|}{ GCC countries } \\
\hline NTM species & $\begin{array}{c}\text { Total number of clinical } \\
\text { isolates }\end{array}$ & Saudi Arabia & Kuwait & Oman & Clinical relevance & Reference \\
\hline M. avium complex & 79 & 68 & 2 & 9 & Pulmonary & {$[17-21]$} \\
\hline M. fortuitum & 90 & 83 & 7 & - & Pulmonary and extrapulmonary & {$[18-21]$} \\
\hline M. simiae & 3 & 2 & - & 1 & Pulmonary & {$[17,19-21]$} \\
\hline M. kansasii & 14 & 12 & 1 & 1 & Pulmonary & {$[17-21]$} \\
\hline M. gordonae & 17 & 16 & 1 & - & Pulmonary and extrapulmonary & {$[18-21]$} \\
\hline M. xenopi & 5 & 5 & - & - & Pulmonary & {$[19-21]$} \\
\hline M. abscessus & 55 & 53 & 2 & - & Pulmonary and extrapulmonary & {$[18-21]$} \\
\hline M. marinum & 2 & 1 & - & 1 & Pulmonary & {$[17,19-21]$} \\
\hline M. chelonae & 9 & 8 & 1 & - & Extrapulmonary & [18-21] \\
\hline M. scrofulaceum & 2 & 2 & - & - & Extrapulmonary & [19-21] \\
\hline M. lentiflavum & 2 & 2 & - & - & Extrapulmonary & [19-21] \\
\hline M. smegmatis & 5 & 5 & - & - & Pulmonary & [19-21] \\
\hline M. szulgai & 3 & 3 & - & - & Pulmonary & {$[19-21]$} \\
\hline M. flavescens & 3 & 2 & - & 1 & Pulmonary & {$[17,19-21]$} \\
\hline M. terrae & 4 & 4 & - & - & Pulmonary & [19-21] \\
\hline M. intracellulare & 9 & 9 & - & - & Pulmonary and extrapulmonary & [19-21] \\
\hline M. gastri & 1 & 1 & - & - & Pulmonary & [19-21] \\
\hline M. asiaticum & 1 & 1 & - & - & Pulmonary & [19-21] \\
\hline M. celatum & 2 & 2 & - & - & Pulmonary & [19-21] \\
\hline Unidentified & 9 & 9 & & & & [19-21] \\
\hline Total & 315 & 290 & 14 & 13 & & \\
\hline
\end{tabular}

our knowledge, this is the first review in scientific reporting aiming to provide an updated insight into the case of NTM particularly in countries of the GCC (Saudi Arabia, Qatar, United Arabs of Emirates (UAE), Kuwait, Bahrain, and Oman) and comparing it with other sites of the world.

\section{Clinical Epidemiology of NTM}

As per the collected data, the most two geographically distributed species of NTM (defined as those reported in most GCC countries) are $M$. avium complex and $M$. kansasii (Table 1) [17-21]. M. avium complex and M. kansasii were found in Saudi Arabia with the uppermost number of reported clinical isolates compared to Kuwait and Oman (Table 1). In fact, the diversity burden of M. avium complex and $M$. kansasii exceeds the Gulf region and covers most regions of the Middle East with a clinical rate of $12.9 \%$ and $9.4 \%$, respectively $[14,17-19,22-29]$.

On the other hand, the two less distributed, but more clinically predominant, NTM species in the GCC countries (defined as those reported highest in clinical occurrence) are $M$. fortuitum, MAC, and M. abscessus (Table 1). $M$. fortuitum and M. abscessus were previously reported in Saudi Arabia and Kuwait, with a similar clinical occurrence in both regions. $M$. fortuitum was accountable for approximately $36 \%$ and $50 \%$ of clinical isolates in Saudi Arabia and Kuwait, respectively. In contrast, $M$. abscessus accounts for at least
21 and 14\% of all reported clinical isolates in Saudi Arabia and Kuwait, respectively (Table 1). Additional NTM species reported from Saudi Arabia include M. gordonae, M. xenopi, M. haemophilum, M. scrofulaceum, M. chelonae, M. smegmatis, and M. szulgai. Although most of these NTM species have been reported with a rare clinical frequency, it is unclear whether their clinical relevance will remain very rare or it will take a different clinical pattern in the coming years. The diversity burden of NTM in Saudi Arabia also comprises a novel species, named $M$. riyadhense [30]. The newly identified kind of mycobacteria, $M$. riyadhense, and $M$. marinum were both reported separately in two independent case studies in Bahrain (Table 2) [31, 32]. Correspondingly, multiple case studies as well as unpublished data from Saudi Arabia are indeed showing a growing trend of $M$. riyadhense, with many isolates being identified recently (Table 2 ).

Nonetheless, as per the data collected from the GCC countries, Saudi Arabia has the highest NTM clinical diversity, with at least 13 highly diversified NTM species. In contrast, in Kuwait and Oman the diversity of clinically reported NTM species reaches to six and four species, respectively. One explanation of this huge difference in the burden of NTM diversity in Saudi Arabia and the rest of Gulf region could be due to a better clinical coverage of NTM in Saudi Arabia compared to the small sample size reported from Kuwait and Oman (14 and 13 clinical isolates, resp.). In line with this explanation, similar diversity trend noted from Saudi Arabia 
TABLE 2: Case studies reported from the GCC countries.

\begin{tabular}{|c|c|c|c|c|}
\hline $\begin{array}{l}\text { Country (number of } \\
\text { cases) }\end{array}$ & Species & Number of cases & Site of infection & Source \\
\hline \multirow{7}{*}{ Saudi Arabia (17) } & M. fortuitum & 4 & $\begin{array}{l}\text { Pulmonary discharges, ascetic fluid, mediastinal infection, peritoneal } \\
\text { dialysis fluid, and lipoid pneumonia. }\end{array}$ & [79-82] \\
\hline & M. abscessus & 4 & $\begin{array}{l}\text { Pulmonary discharge, peritoneal biopsy, peripheral blood, and } \\
\text { permanent catheter tip. }\end{array}$ & [83-86] \\
\hline & M. chelonae & 3 & Blood \& abnormal fluid, breast abscesses, and pleural fluid. & [87-89] \\
\hline & M. marinum & 1 & Wound-elbow. & {$[90]$} \\
\hline & M. kansasii & 1 & Appendiceal abscess. & {$[14]$} \\
\hline & M. szulgai & 1 & Joint aspiration. & [91] \\
\hline & M. riyadhense & 3 & $\begin{array}{c}\text { Maxillary sinus, dural lesion, sclerotic lesions, and pulmonary } \\
\text { infection. }\end{array}$ & {$[30,60,74]$} \\
\hline \multirow{2}{*}{ Qatar (4) } & M. gordonae & 2 & Liver biopsy and urine. & {$[50,51]$} \\
\hline & M. fortuitum & 2 & Myocardial and abdominal abscess. & {$[92,93]$} \\
\hline \multirow{2}{*}{ Bahrain (2) } & M. riyadhense & 1 & Pulmonary discharge. & [32] \\
\hline & M. marinum & 1 & Nasal cavity. & [31] \\
\hline Kuwait (1) & M. abscessus & 1 & Peripheral blood. & [94] \\
\hline
\end{tabular}

has been also reported from neighbors of the GCC countries, constituting other regions of the Middle East, with various extents, such as that reported from Iran (12 species of NTM) and Turkey (18 species of NTM) [28, 29, 33]. Alternatively, Saudi Arabia might be under higher vulnerability to NTM exposure and infection when compared with other GCC countries. However, with this limited amount of reported clinical data from the Gulf region and the little research contribution of UAE, Qatar, and Bahrain, our insights into NTM discrepancies remain largely lacking and necessitate more epidemiological investigations.

\section{Clinical Significance of NTM: Pulmonary and Extrapulmonary Cases}

In Saudi Arabia, 73 out of 95 NTM clinical isolates were reported previously as respiratory cases [19]. This included approximately $76.8 \%$ of all assessed cases between July 2009 and 2010, confirmed mainly in sputum specimen, followed by other sources of specimens such as bronchial wash, lymph node biopsy, and urine [19]. Common pathogens responsible for respiratory cases found in Saudi Arabia were M. fortuitum, M. abscessus, M. kansasii, and MAC [19]. The reported relevance of each pathogen in this study was $34.2 \%, 28.8 \%$, $9.7 \%$, and $6.8 \%$, respectively. Other less clinically relevant pathogens responsible for the reported cases of pulmonary infections include $M$. gordonae, $M$. xenopi, M. celatum, $M$. asiaticum, and $M$. simiae. The rate of definitive pulmonary diseases, which are in agreement with the American Thoracic Society/Infectious Disease Society of America (ATS/IDSA) 2007 guideline, was reported as $67 \%$. Interestingly, $M$. fortuitum also contributed largely to the 24 cases of lung colonizers reported by Varghese et al. (2013). Similar insights have been noted from another study in Saudi Arabia. The study examined respiratory specimens of 142 patients; $28 \%$ of them were diagnosed with definitive pulmonary NTM infections, caused mainly by MAC and M. abscessus [20]. A summary of all respiratory and colonization cases reported so far from Saudi Arabia is outlined in Table 3.

Additionally, Varghese et al. (2013) reported 22 isolates as cases of extrapulmonary NTM infections. Pathogens responsible for these 22 cases include $M$. abscessus, $M$. fortuitum, $M$. intracellulare, M. gordonae, M. lentiflavum, M. scrofulaceum, and $M$. chelonae, with various clinical percentages, ranging from $36.4 \%$ to $4.9 \%$, respectively (Figure 1) [19], wherein $M$. abscessus and M. fortuitum both contributed predominantly to pulmonary and extrapulmonary cases with high clinical occurrence. In Oman, however, MAC, M. simiae, and M. marinum were the main etiological agents of NTM pulmonary diseases (Table 3/Figure 1) [17].

Without any doubt, the high occurrence of reported pulmonary NTM infections that meet the ATS/IDSA criteria in Saudi Arabia and Oman (altogether approximately 40\%) constitute a serious health issue (Table 3 ). It remains unclear, however, whether this is the case only in Saudi Arabia and Oman, or there is a bigger trend of high NTM pulmonary infections covering most countries of the GCC. These health incidences, and suspicions of their bigger geographic burden, should be addressed with great deal of attention by health authorities.

\section{Trends of NTM in Countries of the GCC versus the Rest of the World}

In general, an increase in NTM clinical isolation has been noted from the Gulf region. Such trend is not surprising and has been echoed in other sites of the world including some countries in Europe, Asia, Australia, and America [34-41]. Interestingly, most developing regions situated in Asia (such as South Korea and India) and the Middle East (such as the GCC countries) reported high prevalence of rapidly growing mycobacteria (RGM) in their clinical settings 
TABLe 3: Pulmonary infections caused by NTM in Saudi Arabia and Oman.

\begin{tabular}{|c|c|c|c|c|}
\hline Species & $\begin{array}{c}\text { Number of respiratory } \\
\text { cases }\end{array}$ & $\begin{array}{c}\text { Number of cases meeting the } \\
2007 \text { ATS criteria }\end{array}$ & Number of lung colonizers & References \\
\hline \multicolumn{5}{|c|}{ Saudi Arabia } \\
\hline M. fortuitum & 59 & 17 & 42 & \\
\hline M. abscessus & 45 & 25 & 20 & \\
\hline M. kansasii & 10 & 8 & 2 & \\
\hline MAC & 64 & 27 & 37 & \\
\hline M. gordonae & 11 & 2 & 9 & \\
\hline M. xenopi & 3 & 3 & 0 & \\
\hline M. celatum & 2 & 2 & 0 & \\
\hline M. szulgai & 2 & 2 & 0 & \\
\hline M. asiaticum & 1 & 1 & 0 & \\
\hline M. simiae & 2 & 2 & 0 & \\
\hline Total & 199 & $89(44.7 \%)$ & $110(55.3 \%)$ & {$[19,20]$} \\
\hline \multicolumn{5}{|c|}{ Oman } \\
\hline MAC & 9 & 6 & 3 & \\
\hline M. kansasii & 1 & - & 1 & \\
\hline M. simiae & 1 & 1 & - & \\
\hline M. flavescens & 1 & - & 1 & \\
\hline M. marinum & 1 & 1 & - & \\
\hline Total & 13 & $8(62 \%)$ & $5(38 \%)$ & [17] \\
\hline
\end{tabular}

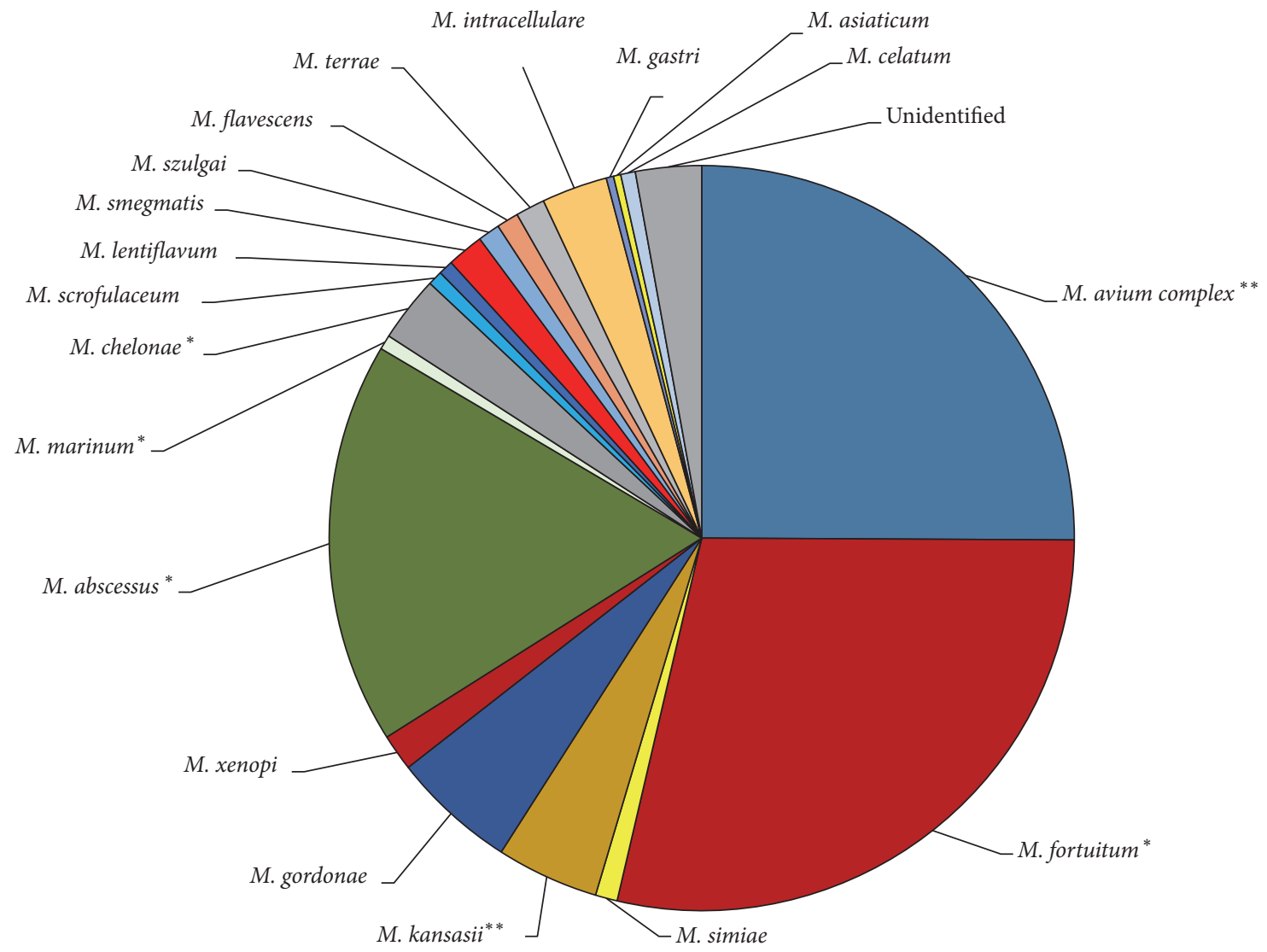

FIGURE 1: Clinical diversity of NTM in the Gulf countries. Illustrating the clinical diversity of NTM in three different GCC countries: Saudi Arabia, Kuwait, and Oman. The $* *$ sign is designated for species reported from Saudi Arabia, Kuwait, and Oman. The $*$ sign, however, indicates that a species is reported from two GCC countries (Saudi Arabia and Oman or Saudi Arabia and Kuwait). Species shown in this figure with (no sign) are only reported from one GCC country. References [17-21]. 


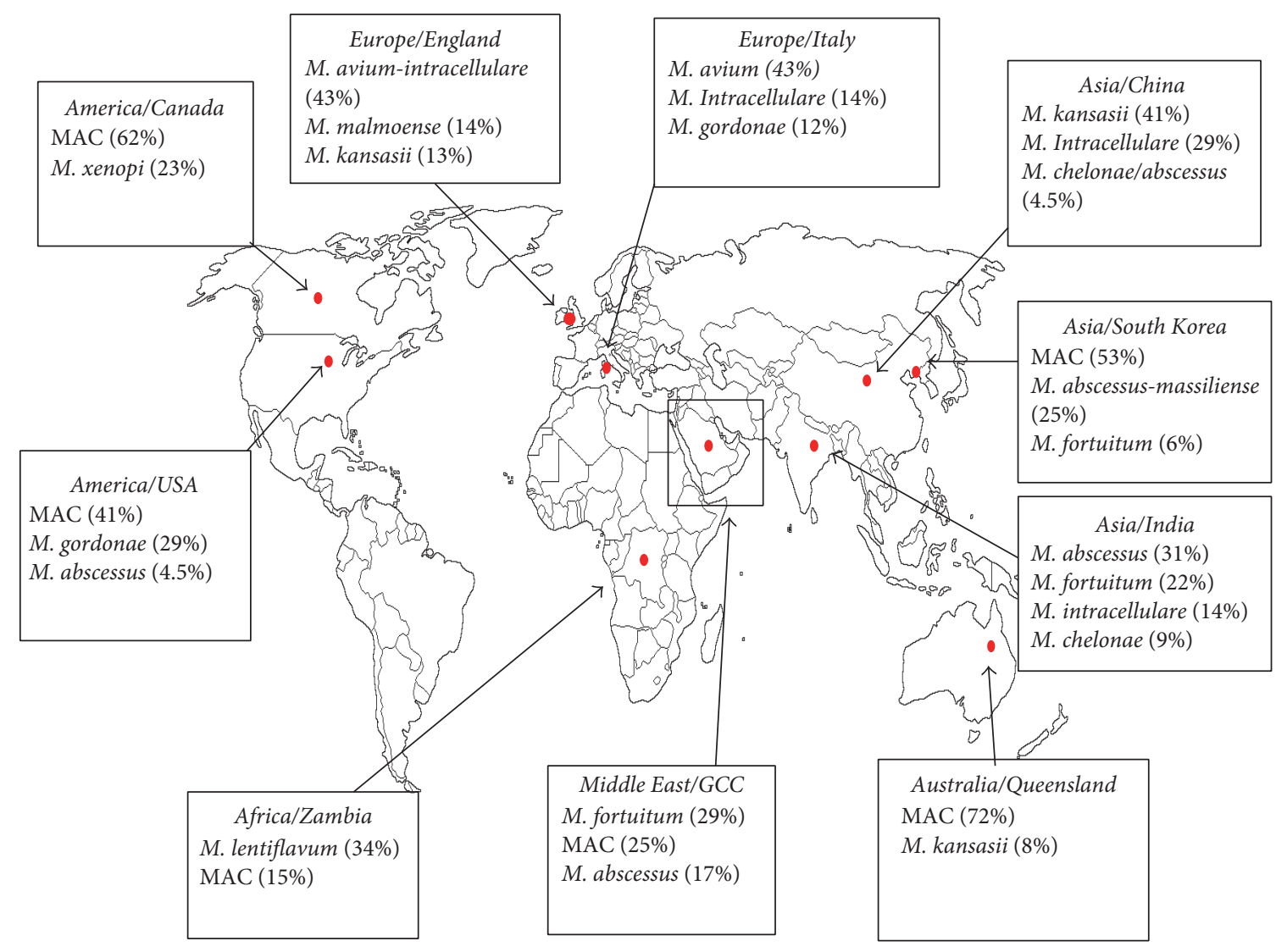

FIGURE 2: Worldwide geographical distribution of NTM. Showing summarized data of NTM identified from human clinical specimens in different geographical settings [19-21, 34-42, 96].

such as M. fortuitum (6-22\%) and M. abscessus (25-31\%) (Figure 2/Table 4) $[38,40]$. These species have been noted from the Gulf region with a clinical relevance of $29 \%$ and $17 \%$, respectively. In contrast, $M$. abscessus has been identified in China and North America with a clinical prevalence of $4.5 \%$ in each case $[36,39]$.

On the other hand, Europe, North America, and Australia showed significant predominance of slowly growing mycobacteria (SGM) among identified clinical isolates (Table 4) [34-37, 41]. The main SGM detected from the above-mentioned regions were MAC (41-43\%), M. kansasii (13-41\%), M. gordonae (12-29\%), and M. malmoense (14\%) [34, 35, 37, 39].

With regard to this notion, it is worth noting that in Europe the clinical isolation of $M$. avium-intracellulare has indeed expanded significantly in the last decade, infecting mainly elderly people [35]. Such profound increase in clinical detection and isolation of MAC species was associated with rapid increase in pulmonary infections [35]. Similar trend has been noted from other sites of Europe, Australia, and North America [34, 36, 37, 41]. Species of MAC were also detected in Asia (India, South Korea, and China), the Middle East (countries of the GCC), and Africa (Zambia) with a clinical rate ranging from 14 to $53 \%[38-40,42]$. On the other hand, other prevalent SGM (including M. kansasii and $M$. gordonae) were evident in the GCC states but with a lower clinical prevalence compared to other industrialized regions.

When it comes to diseases, RGM are usually known to cause cutaneous and osteoarticular infections, while SGM are largely identified as etiological agents for lung and lymph diseases [43]. Thereby, it is rather interesting that in Saudi Arabia $M$. fortuitum and M. abscessus together accounted for $53 \%$ of the total respiratory cases and $47 \%$ of definitive pulmonary infections (Table 3). Similar phenomenon has been noted from India and South Korea.

\section{Environmental Diversity of NTM}

Up to our current time, there is limited number of NTM environmental studies reported from Saudi Arabia and almost no reported environmental isolates from other GCC countries. In Saudi Arabia, Wali et al. (2008) attempted to evaluate the impact of tap water, as a potential source of NTM contamination, on oral cavity of healthy Saudis [44]. The study showed 14/29 isolates as not properly identified and a large proportion of the remaining 15 isolates were identified as M. gordonae (53\%). Additional water contaminants of NTM include M. fortuitum (20\%), M. avium complex (13\%), and M. kansasii (13\%) [44]. Notably, the study highlights the possibility of contaminated tap water supply in the country, 


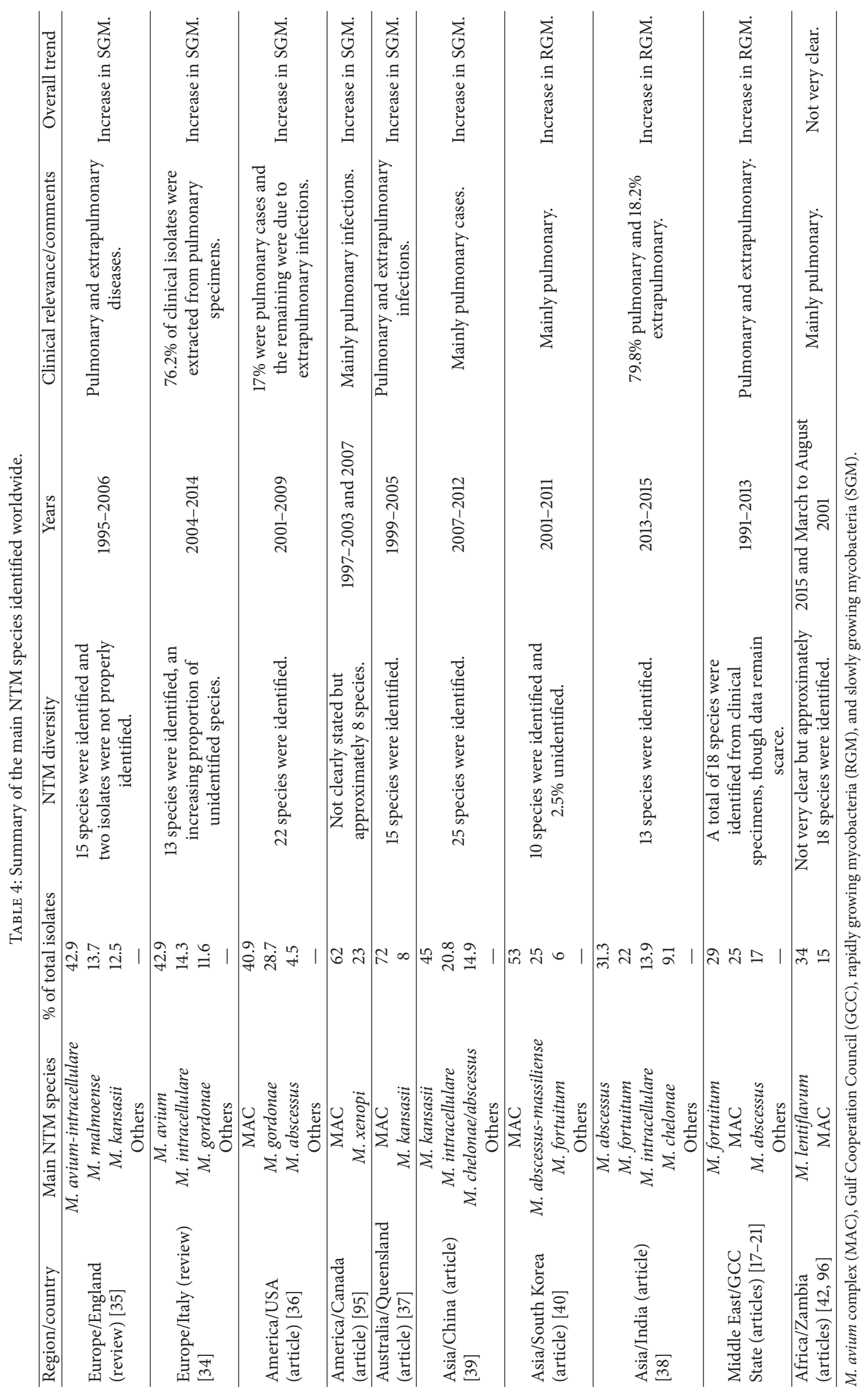


TABLE 5: Environmental NTM species identified in Saudi Arabia.

\begin{tabular}{|c|c|c|c|}
\hline NTM species & Total number of environmental isolates & Source of water contamination & Source \\
\hline M. intracellulare & 5 & Dams & {$[52]$} \\
\hline M. abscessus & 8 & Dams & {$[52]$} \\
\hline M. szulgai & 9 & Dams & {$[52]$} \\
\hline M. fortuitum & 15 & Dams and tap water & {$[44,52]$} \\
\hline M. avium & 14 & Dams and tap water & {$[44,52]$} \\
\hline M. kansasii & 16 & Dams and tap water & {$[44,52]$} \\
\hline M. simiae & 15 & Dams & {$[52]$} \\
\hline M. gordonae & 28 & Dams and tap water & {$[44,52]$} \\
\hline M. terrae complex & 16 & Dams & {$[52]$} \\
\hline M. chelonae & 18 & Dams & {$[52]$} \\
\hline M. malmoense & 21 & Dams & {$[52]$} \\
\hline Unidentified species & 14 & Tap water & {$[44]$} \\
\hline Total & 164 & & \\
\hline
\end{tabular}

especially by $M$. gordonae, which comes in agreement with similar studies reported elsewhere [45-49]. Furthermore, taking into consideration that the clinical relevance of $M$. gordonae was reported not only from Saudi Arabia but also from Kuwait and Qatar, similar issue of water contamination might be worth further investigation in these two regions (as a potential source of $M$. gordonae infections) [18, 50, 51].

Another study from Saudi Arabia has confirmed the contamination of multiple water dams with NTM in Albaha [52]. A total of 13 dams were entitled for the purpose of this study, with almost 520 isolates being collected (between 20 and 30 July of 2013) from multiple environmental resources such as sands, clay, wet stones, and decayed vegetation. Furthermore, a large proportion (79\%) of collected isolates yielded positive results for NTM, which is more than that reported elsewhere [53, 54]. In addition, 145 isolates were carefully selected and subjected to species identification process by Alqumber (2014). Results yielded 11 highly diversified species (ranging from slow to rapid growing mycobacteria). These are as follows: $M$. intracellulare, M. abscessus, $M$. szulgai, M. fortuitum, M. avium, M. kansasii, M. simiae, $M$. gordonae, $M$. terrae complex, $M$. chelonae, and $M$. malmoense (Table 5).

Alarmingly, these dams are heavily used for human consumption, water recreation, and agriculture use, and accordingly inhabitants of the city (Albaha) are under an accelerating risk for NTM exposure and infection, especially those with immunological diseases. This calls for the relevant health authorities' attention to tackle the issue properly and promptly to reduce exposure to NTM in contaminated water resources. In addition, the high diversity of reported NTM species from water dams in Saudi Arabia supports the notion that water resources can be the main facilitator of NTM exposure and infections in arid and desert climates, such as that seen in Saudi Arabia and other Gulf countries.

Comparable spectrum of NTM species was reported from other regions of the Middles East and neighbors of the GCC countries, including Iran, Turkey, and Iraq. In Iraq, $M$. chelonae was one of the most common NTM environmental species (18\%), isolated from tap water, followed by M. avium complex and M. fortuitum (Figure 3) [55-57]. The latter two species were also isolated from milk powder and horses feces, respectively [55-57]. Environmental isolates reported from Turkey, however (including M. lentiflavum, M. gordonae, and M. peregrinum), were mainly isolated from hospital water supply [58]. Overall, comprehensive reviews published from Europe, Australia, and Asia as well as the Middle East come in favor of increased rate of pulmonary infections largely caused by MAC species, the most predominant SGM species worldwide, due to contaminated water supply $[9,34,35,37$, 59]. The scale of the problem, however, remains to be fully defined and characterized in each country of the GCC for more accurate picture.

\section{Challenges in Diagnosing NTM Diseases}

Diagnosing mycobacterial infections entitles three different stages (clinical, radiological, and microbiological examinations) as per the ATS/IDSA-2007 guideline [8]. In the GCC countries, however, several challenges have been noted during the clinical and microbiological phases. These challenges were addressed accordingly in the following relative sections.

6.1. The Need for Optimized Local Diagnostic Criteria. A rapid and accurate recognition of NTM clinical symptoms is indeed a much-desired goal but nonetheless a very challenging one in the Gulf region, not only due to the high diversity of clinical symptoms for each NTM infection or their overlapping features with other diseases, but also due to scarce epidemiological data and the absence of locally optimized diagnostic criteria. The latter two issues make improper identification of NTM a persisting challenge in the Gulf region, as evident from several case studies $[30,60]$. Thus, to ensure a prompt recognition of NTM cases in this geographic region, the NTM diagnostic criteria (ATS/IDSA, 2007) should be tested, similar to what has been done elsewhere, and accordingly optimized [61]. This is especially imperative since not all NTM species relevant in countries of the GCC are represented in the ATS/IDSA 2007 criteria. A good example of this is the newly emerging species of $M$. riyadhense. While 


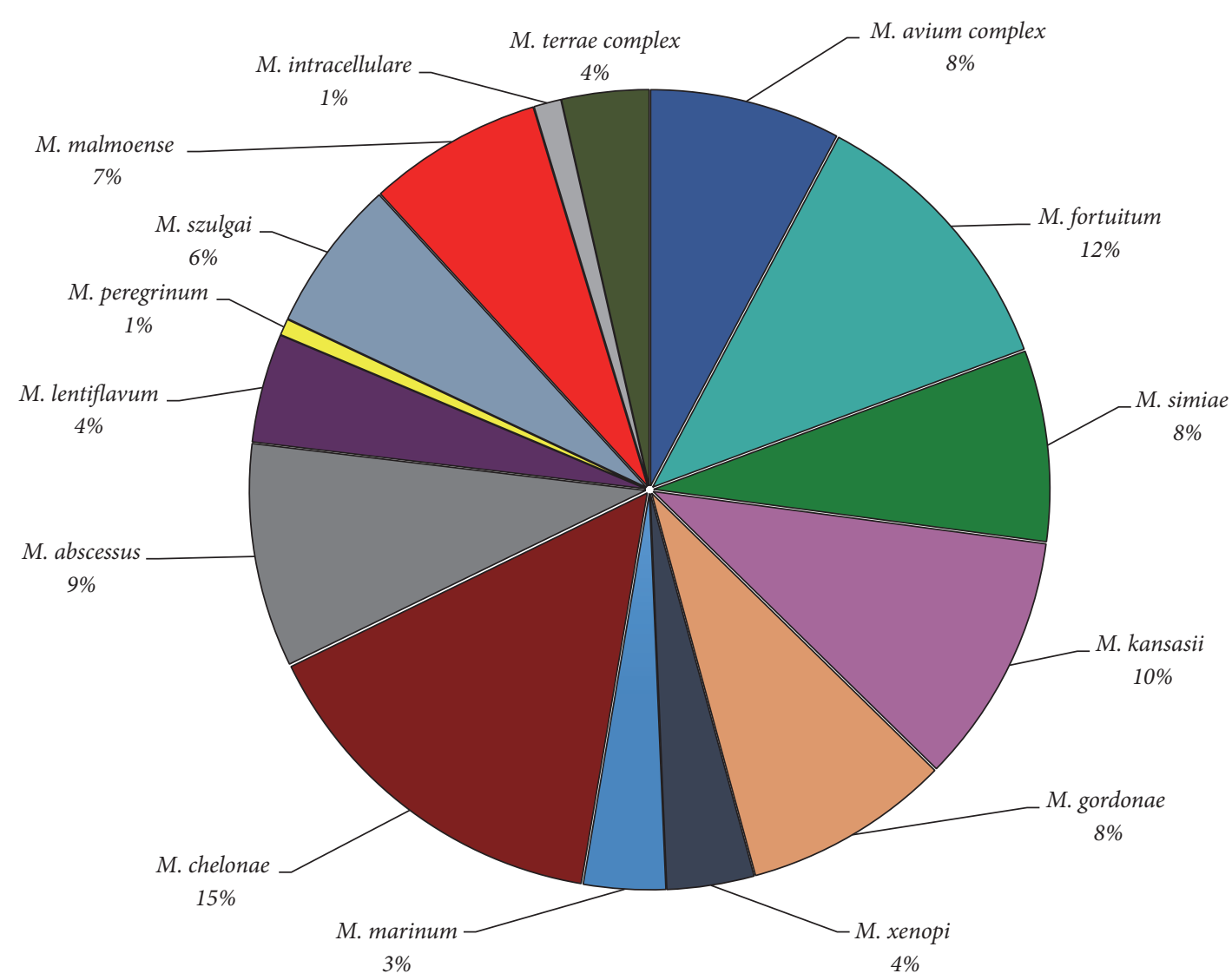

FIGURE 3: Environmental isolates reported from Saudi Arabia, Iraq, and Turkey. Illustrating the environmental diversity of NTM in Saudi Arabia, Iraq, and Turkey. Species' names and their relevant environmental percentages are also illustrated. References [44, 52, 55-58].

this species has increased in occurrence during the last years, however, its clinical identification remains a challenge. This emphasizes the need for optimized diagnostic criteria in the region, as well as more epidemiological data from the Gulf countries.

6.2. Cross-Contamination and Pseudoinfection. Due to the ambiguity of NTM clinical symptoms, diagnosing NTM diseases relies heavily on the accuracy of laboratories test results. Several factors, however, might undermine the accuracy of laboratories test results and accelerate the frequency of false-positive outcome in the Gulf region. The most two identified factors are (1) cross-contamination and (2) pseudoinfection. Although cross-contamination rate is considered infrequent in most specialized laboratories worldwide, and in some cases inevitable due to human error with a low rate of $3 \%$, it might take place profoundly at belowstandards NTM/TB laboratories [62-64]. This issue has been addressed previously concerning some laboratories in the Gulf region [64]. Furthermore, in Saudi Arabia, 22 cases of cross-contamination have been identified through the use of molecular identification techniques, wherein the source of contamination was traced back to a common contaminated buffer [65]. In addition, based on an earlier personal visit to the main TB diagnostic laboratories in Saudi Arabia, in 2004, two main conclusive remarks have been declared
[64]. The visited laboratories in Saudi Arabia displayed (1) inadequate laboratories equipment combined with high load of specimens handling and (2) laboratories personnel showed confusion between cross-contamination and other forms of contamination (such as fungus and bacterial contamination) [64]. This raises further speculations of a much bigger relevance of cross-contamination in the country. In addition, better-trained personnel and establishing a standard laboratory protocols were selected as the resolving actions for crosscontamination in countries of the GCC.

On the other hand, unlike the case of crosscontamination, pseudoinfection is more common worldwide and causes a real problem in the issue of accurate NTM diagnosis. Pseudoinfection relies mainly on the resistance capabilities of NTM species towards most disinfectants. This explains their ability to survive on medical devices and laboratory tools used in the diagnosis procedure (such as bronchoscopes) as well as unsterile patients' samples (such as expectorated sputum), causing false-positive NTM diagnosis. Similar cases of pseudoinfection were reported previously in the literature [66]. So far, there is a limited amount of reported cases of pseudoinfection from the Gulf region. It is unclear whether this is due to the scarce amount of available data or due to the insignificant nature of the issue. Furthermore, the 2007 ATS/IDSA guideline offers a practical solution to differentiate pseudoinfection from real NTM 
diseases. Nonetheless, a better surveillance system must be established to ensure the compliance of physicians and laboratories' technicians with these rules and regulations, especially in local diagnostic laboratories. This may decrease rates of NTM misdiagnosis in the Gulf region and ensure, to some extent, accurate and effective treatments.

\section{Pathogenicity and Common Risk Factors}

Increased predisposition of genetic factors as well as undergoing chronic lung and immune diseases put patients at higher risk of developing NTM infections. In Saudi Arabia, the majority of pulmonary and extrapulmonary cases, reported by Varghese et al. (2013), were observed in patients with previous history of $M$. tuberculosis diseases (PMTD), chronic obstructive pulmonary disorders (COPD), HIV, and cystic fibrosis. The predominant risk factor observed in this study was PMTD (29.5\%). In addition, it appears that elderly men in Saudi Arabia are more susceptible to NTM infections as correspondingly reported from another GCC country [18, 19].

In Oman, however, three out of 13 cases have a risk factor to NTM infections. This was due to either heart diseases, HIV, or PMTD, with the same clinical relevance reported for each factor. Thus, the high number of living immunosuppressive patients in the Middle East, comprised of 500,000 people, as well as the high consanguinity rate in some Gulf countries (around 60\%), could lead to a drastic increase in NTM infections in the future $[67,68]$.

While it is true that NTM infections occur predominantly in immunocompromised patients, contradictory cases were also reported from the Gulf region. For instance, pulmonary infections that are caused by M. abscessus usually occur to those with genetic predisposition, immunosuppressive, and chronic lung diseases $[69,70]$. However, in Saudi Arabia, two healthy individuals, with no genetic and physiological risk factors, were infected with chronic lung diseases caused by $M$. abscessus [71]. These two cases were incorrectly diagnosed as TB before accurate molecular identification. Also, in Kuwait, M. abscessus outbreak was responsible for 4 cases of severe infections in a pediatric emergency unit [72].

Notably, the existence of hypervirulent stains of $M$. abscessus in countries of the GCC, compared to the rest of the world, has not been reported before and, in fact, might have been overlooked. To further understand the impact of strain's variation on pathogenicity and drug resistance in countries of the GCC, more research is needed. This should impact positively on the clinical side and may revolutionize the health care system in the region towards precision medicine.

\section{Emergence of New Species of NTM: M. riyadhense as an Example}

The debate of newly emergent NTM species has always been linked to recent advancements in fingerprinting and identification techniques. Among many cases of newly identified NTM species, $M$. riyadhense is the only kind that was initially isolated from Saudi Arabia. It was first encountered in a 19year-old male with a bone infection in left maxillary sinus and with no signs of destructive lung structure. This case was suspected to be bone TB and accordingly the patient was treated with antituberculosis treatment [30]. Later it was found that there are shared genetic virulent factors in esat-6 and cfp-10 genes between $M$. tuberculosis and $M$. riyadhense, with limited understanding to their impact on $M$. riyadhense pathogenicity. Furthermore, the clinical diversity burden of $M$. riyadhense sparked globally after its successful discovery, indicating that it is not geographically contained in Saudi Arabia. It was reported to cause cases of pulmonary infections in Bahrain, France, and Korea as well as three other cases reported recently from Saudi Arabia $[60,73,74]$. Similar to the initial case of $M$. riyadhense, identification of this species was only available through DNA sequencing of certain conserved genes. This underlines the importance of the introduction of $M$. riyadhense in the commercial lineprobe assays to ease the rapid identification of $M$. riyadhense and reduce health implications related to late diagnosis worldwide [75]. As shown in the case of $M$. riyadhense, a small percentage of genetic variance (as low as $0.1 \%$ ) in DNA alignment of well-conserved genes might elucidate the presence of a novel species.

Meanwhile the role of microevolution on bringing about new NTM species cannot be overruled. For instance, evidence of microevolution events was previously reported in a patient infected with TB in the GCC region [76]. In this case study, clonal $M$. tuberculosis variants were identified from a total of five body isolates collected from the same patient: three of them originated from the same site (urine) [76]. Although several studies, published elsewhere, have reported different cases of mixed-TB infections in the same patient, suborgan compartmentalization was rarely reported [77, 78]. It has been proposed by Al-Hajoj et al. (2010) that the long period of infection (associated with a higher rate of mutations) and perhaps genetic drift were the major contributors for the emergence of a subclonal $M$. tuberculosis variant in this patient. Thus, the possibility of similar microevolutionary events, under similar circumstances, in NTM cases should not be entirely excluded. However, there is a trivial research interest on this area in the Gulf region, largely justified by the little amount of solid evidence supporting this theory in encountered clinical cases. In addition, this idea is largely overlooked in NTM research due to assumptions of its small clinical value and accordingly such type of research is unlikely to be supported.

\section{Conclusion}

This review aimed to address the main NTM aspects and challenges in the GCC countries. Although the reported data from the GCC is limited in terms of sample size (most reported papers do not have a nationwide sample size) and geographic coverage (not all countries within the GCC state contribute to the reported data in the literature) it is associated with so far an increasing trend of NTM clinical diversity and severity of infections, with high rate of definitive respiratory diseases. However, more research is required to fill in the recognizable gaps that clearly exist in the literature regarding NTM in countries of the GCC. 


\section{Competing Interests}

The authors declare that there is no conflict of interests regarding the publication of this paper.

\section{References}

[1] M. Ventura, C. Canchaya, A. Tauch et al., "Genomics of Actinobacteria: tracing the evolutionary history of an ancient phylum," Microbiology and Molecular Biology Reviews, vol. 71, no. 3, pp. 495-548, 2007.

[2] M. M. Johnson and J. A. Odell, "Nontuberculous mycobacterial pulmonary infections," Journal of Thoracic Disease, vol. 6, no. 3, pp. 210-220, 2014.

[3] E. Tortoli, "Impact of genotypic studies on mycobacterial taxonomy: the new mycobacteria of the 1990s," Clinical Microbiology Reviews, vol. 16, no. 2, pp. 319-354, 2003.

[4] World Health Organisation, Global Tuberculosis Report 2015, World Health Organisation, Geneva, Switzerland, 2015.

[5] E. C. Hett and E. J. Rubin, "Bacterial growth and cell division: a mycobacterial perspective," Microbiology and Molecular Biology Reviews, vol. 72, no. 1, pp. 126-156, 2008.

[6] J. Van Ingen, M. J. Boeree, D. Van Soolingen, and J. W. Mouton, "Resistance mechanisms and drug susceptibility testing of nontuberculous mycobacteria," Drug Resistance Updates, vol. 15, no. 3, pp. 149-161, 2012.

[7] S. Faria, I. Joao, and L. Jordao, "General overview on nontuberculous mycobacteria, biofilms, and human infection," Journal of Pathogens, vol. 2015, Article ID 809014, 10 pages, 2015.

[8] D. E. Griffith, T. Aksamit, B. A. Brown-Elliott et al., "An official ATS/IDSA statement: diagnosis, treatment, and prevention of nontuberculous mycobacterial diseases," American Journal of Respiratory and Critical Care Medicine, vol. 175, no. 4, pp. 367416, 2007.

[9] S. Simons, J. van Ingen, P.-R. Hsueh et al., "Nontuberculous mycobacteria in respiratory tract infections, Eastern Asia," Emerging Infectious Diseases, vol. 17, no. 3, pp. 343-349, 2011.

[10] E. E. Bodle, J. A. Cunningham, P. Della-Latta, N. W. Schluger, and L. Saiman, "Epidemiology of nontuberculous mycobacteria in patients without HIV infection, New York City," Emerging Infectious Diseases, vol. 14, no. 3, pp. 390-396, 2008.

[11] I. K. Neonakis, Z. Gitti, I. S. Kourbeti et al., "Mycobacterial species diversity at a general hospital on the island of crete: first detection of mycobacterium lentiflavum in Greece," Scandinavian Journal of Infectious Diseases, vol. 39, no. 10, pp. 875-879, 2007.

[12] P. M. Cassidy, K. Hedberg, A. Saulson, E. McNelly, and K. L. Winthrop, "Nontuberculous mycobacterial disease prevalence and risk factors: a changing epidemiology," Clinical Infectious Diseases, vol. 49, no. 12, pp. e124-e129, 2009.

[13] M. A. De Groote and G. Huitt, "Infections due to rapidly growing mycobacteria," Clinical Infectious Diseases, vol. 42, no. 12, pp. 1756-1763, 2006.

[14] M. A. Enani, H. H. Frayha, and M. A. Halim, "An appendiceal abscess due to Mycobacterium kansasii in a child with AIDS," Clinical Infectious Diseases, vol. 27, no. 4, pp. 891-892, 1998.

[15] M. E. Ellis and S. M. H. Qadri, "Mycobacteria other than tuberculosis producing disease in a tertiary referral hospital," Annals of Saudi Medicine, vol. 13, no. 6, pp. 508-515, 1993.

[16] P. Chetchotisakd, S. Kiertiburanakul, P. Mootsikapun, S. Assanasen, R. Chaiwarith, and S. Anunnatsiri, "Disseminated nontuberculous mycobacterial infection in patients who are not infected with HIV in Thailand," Clinical Infectious Diseases, vol. 45, no. 4, pp. 421-427, 2007.

[17] S. H. Al-Mahruqi, J. Van Ingen, S. Al-Busaidy et al., "Clinical relevance of nontuberculous mycobacteria, Oman," Emerging Infectious Diseases, vol. 15, no. 2, 2009.

[18] E. Mokaddas and S. Ahmad, "Species spectrum of nontuberculous mycobacteria isolated from clinical specimens in Kuwait," Current Microbiology, vol. 56, no. 5, pp. 413-417, 2008.

[19] B. Varghese, Z. Memish, N. Abuljadayel, R. Al-Hakeem, F. Alrabiah, and S. A. Al-Hajoj, "Emergence of clinically relevant non-tuberculous mycobacterial infections in Saudi Arabia," PLoS Neglected Tropical Diseases, vol. 7, no. 5, Article ID e2234, 2013.

[20] A. Al-Harbi, H. Al-Jahdali, S. Al-Johani, S. Baharoon, S. Bin Salih, and M. Khan, "Frequency and clinical significance of respiratory isolates of non-tuberculous mycobacteria in Riyadh, Saudi Arabia," Clinical Respiratory Journal, vol. 10, no. 2, pp. 198-203, 2016.

[21] R. Zaman, "Tuberculosis in Saudi Arabia: epidemiology and incidence of Mycobacterium tuberculosis and other mycobacteriai species," Tubercle, vol. 72, no. 1, pp. 43-49, 1991.

[22] R. Pinto-Powell, K. N. Olivier, B. J. Marsh et al., "Skin testing with Mycobacterium avium sensitin to identify infection with M. avium complex in patients with cystic fibrosis," Clinical Infectious Diseases, vol. 22, no. 3, pp. 560-562, 1996.

[23] F. M. T. Lewis, B. J. Marsh, and C. F. Von Reyn, "Fish tank exposure and cutaneous infections due to mycobacterium marinum: tuberculin skin testing, treatment, and prevention," Clinical Infectious Diseases, vol. 37, no. 3, pp. 390-397, 2003.

[24] R. N. Hamam, B. Noureddin, H. I. Salti, R. Haddad, and J. M. Khoury, "Recalcitrant post-LASIK Mycobacterium chelonae keratitis eradicated after the use of fourth-generation fluoroquinolone," Ophthalmology, vol. 113, no. 6, pp. 950-954, 2006.

[25] M. M. Balkis, M. M. Kattar, G. F. Araj, and S. S. Kanj, "Fatal disseminated Mycobacterium simiae infection in a non-HIV patient," International Journal of Infectious Diseases, vol. 13, no. 5, pp. e286-e287, 2009.

[26] M. A. El-Khalawany, "Atypical mycobacterial cutaneous infections in Egyptians: a clinicopathological study," Journal of Dermatology, vol. 41, no. 4, pp. 303-310, 2014.

[27] G. Bamias, G. L. Daikos, S. I. Siakavellas et al., "Atypical mycobacterial infection presenting as persistent skin lesion in a patient with ulcerative colitis," Case Reports in Medicine, vol. 2011, Article ID 480987, 4 pages, 2011.

[28] M. Gunaydin, K. Yanik, C. Eroglu et al., "Distribution of Nontuberculous Mycobacteria strains," Annals of Clinical Microbiology and Antimicrobials, vol. 12, 2013.

[29] A. A. Velayati, S. Rahideh, Z. Derakhshani Nezhad, P. Farnia, and M. Mirsaeidi, "Nontuberculous mycobacteria in Middle East: current situation and future challenges," International Journal of Mycobacteriology, vol. 4, no. 1, pp. 7-17, 2015.

[30] J. van Ingen, S. A. M. Al-Haijoj, M. Boeree et al., "Mycobacterium riyadhense sp. nov., a non-tuberculous species identified as Mycobacterium tuberculosis complex by a commercial lineprobe assay," International Journal of Systematic and Evolutionary Microbiology, vol. 59, no. 5, pp. 1049-1053, 2009.

[31] S. T. Alkhawaja and N. N. Khalifa, "Mycobacterium marinum infection after infliximab therapy," Iranian Journal of Allergy, Asthma and Immunology, vol. 9, pp. 255-257, 2010. 
[32] S. Godreuil, H. Marchandin, A.-L. Michon et al., "Mycobacterium riyadhense pulmonary infection, France and Bahrain," Emerging Infectious Diseases, vol. 18, no. 1, pp. 176-178, 2012.

[33] N. Albayrak, H. ŞimşEk, F. Sezen, A. ArslantÜrk, C. Tarhan, and I. Ceyhan, "[Evaluation of the distribution of non-tuberculous mycobacteria strains isolated in national tuberculosis reference laboratory in 2009-2010, Turkey]," Mikrobiyoloji Bulteni, vol. 46, no. 4, pp. 560-567, 2012.

[34] L. Rindi and C. Garzelli, "Increase in non-tuberculous mycobacteria isolated from humans in Tuscany, Italy, from 2004 to 2014," BMC Infectious Diseases, vol. 16, article 44, 2016.

[35] J. E. Moore, M. E. Kruijshaar, L. P. Ormerod, F. Drobniewski, and I. Abubakar, "Increasing reports of non-tuberculous mycobacteria in England, Wales and Northern Ireland, 19952006," BMC Public Health, vol. 10, article no. 612, 2010.

[36] G. Satyanarayana, S. K. Heysell, K. W. Scully, and E. R. Houpt, "Mycobacterial infections in a large Virginia hospital, 20012009," BMC Infectious Diseases, vol. 11, article 113, 2011.

[37] R. M. Thomson, "Changing epidemiology of pulmonary nontuberculous mycobacteria infections," Emerging Infectious Diseases, vol. 16, no. 10, pp. 1576-1583, 2010.

[38] J. Umrao, D. Singh, A. Zia et al., "Prevalence and species spectrum of both pulmonary and extrapulmonary nontuberculous mycobacteria isolates at a tertiary care center," International Journal of Mycobacteriology, vol. 5, no. 3, pp. 288-293, 2016.

[39] J. Wu, Y. Zhang, J. Li et al., "Increase in nontuberculous mycobacteria isolated in Shanghai, China: results from a population-based study," PLoS ONE, vol. 9, no. 10, Article ID e109736, 2014.

[40] S. W. Ryoo, S. Shin, M.-S. Shim et al., "Spread of nontuberculous mycobacteria from 1993 to 2006 in Koreans," Journal of Clinical Laboratory Analysis, vol. 22, no. 6, pp. 415-420, 2008.

[41] M. Al Houqani, F. Jamieson, P. Chedore, M. Mehta, K. May, and T. K. Marras, "Isolation prevalence of pulmonary nontuberculous mycobacteria in Ontario in 2007," Canadian Respiratory Journal, vol. 18, no. 1, pp. 19-24, 2011.

[42] P. Chanda-Kapata, N. Kapata, E. Klinkenberg et al., "Nontuberculous mycobacteria (NTM) in Zambia: prevalence, clinical, radiological and microbiological characteristics," $B M C$ Infectious Diseases, vol. 15, no. 1, article 500, 2015.

[43] E. Tortoli, "Clinical manifestations of nontuberculous mycobacteria infections," Clinical Microbiology and Infection, vol. 15, no. 10, pp. 906-910, 2009.

[44] S. Wali, M. Abdelaziz, A. Krayem et al., "The presence of atypical mycobacteria in the mouthwashes of normal subjects: role of tap water and oral hygiene," Annals of Thoracic Medicine, vol. 3, no. 1, pp. 5-8, 2008.

[45] B. L. Robinson, S. Grzybowski, E. J. Bowmer, J. McDiarmid, E. I. Whittaker, and K. Tanner, "Atypical mycobacterial disease in British Columbia, 1960-1967," Canadian Medical Association journal, vol. 101, no. 1, pp. 17-24, 1969.

[46] P. M. Arnow, M. Bakir, K. Thompson, and J. L. Bova, "Endemic contamination of clinical specimens by Mycobacterium gordonae," Clinical Infectious Diseases, vol. 31, no. 2, pp. 472-476, 2000.

[47] P. A. Jenkins, "The epidemiology of opportunist mycobacterial infections in Wales, 1952-1978," Reviews of Infectious Diseases, vol. 3, no. 5, pp. 1021-1023, 1981.

[48] M. Debrunner, M. Salfinger, O. Brändli, and A. Von Graevenitz, "Epidemiology and clinical significance of nontuberculous mycobacteria in patients negative for human immunodeficiency virus in switzerland," Clinical Infectious Diseases, vol. 15, no. 2, pp. 330-345, 1992.

[49] J. O. Falkinham III, "Nontuberculous mycobacteria in the environment," Clinics in Chest Medicine, vol. 23, no. 3, pp. 529$551,2002$.

[50] L. N. Jarikre, "Case report: disseminated Mycobacterium gordonae infection in a nonimmunocompromised host," The American Journal of the Medical Sciences, vol. 302, no. 6, pp. 382-384, 1991.

[51] L. N. Jarikre, "Mycobacterium gordonae genitourinary disease," Genitourinary Medicine, vol. 68, no. 1, pp. 45-46, 1992.

[52] M. A. Alqumber, "Prevalence of mycobacteria in water reservoirs of Albaha, Saudi Arabia," Saudi Medical Journal, vol. 35, no. 5, pp. 466-471, 2014.

[53] I. Perez-Martinez, D. A. Aguilar-Ayala, E. Fernandez-Rendon et al., "Occurrence of potentially pathogenic nontuberculous mycobacteria in Mexican household potable water: a pilot study," BMC Research Notes, vol. 6, no. 1, article no. 531, 2013.

[54] R. M. Thomson, R. Carter, C. Tolson, C. Coulter, F. Huygens, and M. Hargreaves, "Factors associated with the isolation of Nontuberculous mycobacteria (NTM) from a large municipal water system in Brisbane, Australia," BMC Microbiology, vol. 13, no. 1, article no. 89, 2013.

[55] A. A. Al-Sulami, A. M. R. Al-Taee, and Q. H. Wida'a, "Isolation and identification of Mycobacterium avium complex and other nontuberculosis mycobacteria from drinking-water in Basra governorate, Iraq," Eastern Mediterranean Health Journal, vol. 18, no. 3, pp. 274-278, 2012.

[56] K. I. Hassan and A. A. Ali, "Detection of Mycobacterium avium in milk powder using species specific PCR," International Journal of Advanced Science and Engineering Technology On Line, vol. 2, no. 2, pp. 115-119, 2012.

[57] A. M. Hamzah, "Mycobacterium spp. isolated from horses fecal samples," International Journal of Medicine and Pharmaceutical Sciences (IJMPS), vol. 3, no. 3, pp. 23-28, 2013.

[58] G. E. Genc, E. Richter, and Z. Erturan, "Isolation of nontuberculous mycobacteria from hospital waters in Turkey," APMIS, vol. 121, no. 12, pp. 1192-1197, 2013.

[59] G. Satta, T. D. McHugh, J. Mountford, I. Abubakar, and M. Lipman, "Managing pulmonary nontuberculous mycobacterial infection. time for a patient-centered approach," Annals of the American Thoracic Society, vol. 11, no. 1, pp. 117-121, 2014.

[60] M. M. Saad, A. N. Alshukairi, M. O. Qutub, N. A. Elkhizzi, H. M. Hilluru, and A. S. Omrani, "Mycobacterium riyadhense infections," Saudi Medical Journal, vol. 36, no. 5, pp. 620-625, 2015.

[61] D. Chae, "The impact of a new ATS/IDSA guideline for nontuberculous mycobacterial disease on the diagnosis of nontuberculous mycobacterial lung disease in Korea," Chest, vol. 138, no. 4, p. 681A, 2010.

[62] M. D. C. Ramos, H. Soini, G. C. Roscanni, M. Jaques, M. C. Villares, and J. M. Musser, "Extensive cross-contamination of specimens with Mycobacterium tuberculosis in a reference laboratory," Journal of Clinical Microbiology, vol. 37, no. 4, pp. 916-919, 1999.

[63] S. Segal-Maurer, B. N. Kreiswirth, J. M. Burns et al., "Mycobacterium tuberculosis specimen contamination revisited: the role of laboratory environmental control in a pseudo-outbreak," Infection Control and Hospital Epidemiology, vol. 19, no. 2, pp. 101-105, 1998. 
[64] S. A. Al-Hajoj and F. A. Alrabiah, "Role of tuberculosis laboratories in Saudi Arabia. A call to implement standardized procedures," Saudi Medical Journal, vol. 25, no. 11, pp. 1545$1548,2004$.

[65] S. A. Al-Hajoj, "Molecular strain typing of Mycobacterium tuberculosis isolates to detect cross-contamination events: proposed modifications to prevent its recurrence," Saudi Medical Journal, vol. 30, no. 12, pp. 1515-1519, 2009.

[66] R. Weinstein and W. Stamm, "Pseudoepidemics in hospital," The Lancet, vol. 310, no. 8043, pp. 862-864, 1977.

[67] S. K. Field, D. Fisher, and R. L. Cowie, "Mycobacterium avium complex pulmonary disease in patients without HIV infection," Chest, vol. 126, no. 2, pp. 566-581, 2004.

[68] M. I. El Mouzan, A. A. Al Salloum, A. S. Al Herbish, M. M. Qurachi, and A. A. Al Omar, "Consanguinity and major genetic disorders in Saudi children: a community-based cross-sectional study," Annals of Saudi Medicine, vol. 28, no. 3, pp. 169-173, 2008.

[69] W.-J. Koh, O. J. Kwon, and K. S. Lee, "Diagnosis and treatment of nontuberculous mycobacterial pulmonary diseases: a Korean perspective," Journal of Korean Medical Science, vol. 20, no. 6, pp. 913-925, 2005.

[70] C. L. Daley and D. E. Griffith, "Pulmonary disease caused by rapidly growing mycobacteria," Clinics in Chest Medicine, vol. 23, no. 3, pp. 623-632, 2002.

[71] B. Varghese, S. E. Shajan, M. O. Al Saedi, and S. A. Al-Hajoj, "First case report of chronic pulmonary lung disease caused by Mycobacterium abscessus in two immunocompetent patients in Saudi Arabia," Annals of Saudi Medicine, vol. 32, no. 3, pp. 312-314, 2012.

[72] W. Jamal, M. F. Salama, G. Al Hashem et al., "An outbreak of Mycobacterium abscessus infection in a Pediatric Intensive Care Unit in Kuwait," Pediatric Infectious Disease Journal, vol. 33, no. 3, pp. e67-e70, 2014.

[73] J.-I. Choi, J.-H. Lim, S.-R. Kim et al., "Lung infection caused by Mycobacterium riyadhense confused with Mycobacterium tuberculosis: the first case in Korea," Annals of Laboratory Medicine, vol. 32, no. 4, pp. 298-303, 2012.

[74] M. Garbati and A. Hakawi, "Mycobacterium riyadhense lung infection in a patient with HIV/AIDS," Sub-Saharan African Journal of Medicine, vol. 1, no. 1, pp. 56-58, 2014.

[75] S. Al-Hajoj, B. Varghese, J. van Ingen, and D. van Soolingen, "Mycobacterium riyadhense overlooked: we can only find what we are looking for," Journal of Infection in Developing Countries, vol. 7, no. 3, pp. 293-294, 2013.

[76] S. A. M. Al-Hajoj, O. Akkerman, I. Parwati et al., "Microevolution of Mycobacterium tuberculosis in a tuberculosis patient," Journal of Clinical Microbiology, vol. 48, no. 10, pp. 3813-3816, 2010.

[77] D. V. D. García, M. Marín, S. Andrés, G. Lorenzo, M. J. Ruiz-Serrano, and E. Bouza, "Complex clonal features in an Mycobacterium tuberculosis infection in a two-year-old child," The Pediatric Infectious Disease Journal, vol. 25, no. 5, pp. 457459, 2006.

[78] I. C. Shamputa, L. Jugheli, N. Sadradze et al., "Mixed infection and clonal representativeness of a single sputum sample in tuberculosis patients from a penitentiary hospital in Georgia," Respiratory Research, vol. 7, article 99, 2006.

[79] M. A. Simbli, F. A. Niaz, and J. S. Al-Wakeel, "Encapsulating peritoneal sclerosis in a peritoneal dialysis patient presenting with complicated Mycobacterium fortuitum peritonitis," Saudi
Journal of Kidney Diseases and Transplantation, vol. 23, no. 3, pp. 635-641, 2012.

[80] A. U. Syed, R. Hussain, A. N. Bhat et al., "Mediastinitis due to Mycobacterium fortuitum infection following Fontan operation in a child," Scandinavian Cardiovascular Journal, vol. 31, no. 5, pp. 311-316, 1997.

[81] S. H. Annobil, G. A. Jamjoom, R. Bobo, and J. Iyengar, "Fatal lipoid pneumonia in an infant complicated by Mycobacterium fortuitum infection," Tropical and Geographical Medicine, vol. 44, no. 1-2, pp. 160-164, 1992.

[82] F. M. Al Majid, "Peritonitis due to Mycobacterium fortuitum following gastric banding," Saudi Journal of Gastroenterology, vol. 16, no. 2, pp. 113-115, 2010.

[83] A.-W. N. Meshikhes, A. Al-Gassab, L. Y. Al-Jaffar, M. Tinguria, Z. S. Al-Meer, and F. Borgio, "Atypical mycobacteria: an unusual cause of breast abscess," Annals of Saudi Medicine, vol. 17, no. 3 , pp. 337-339, 1997.

[84] N. Siddiqi and I. Sheikh, "Peritonitis caused by Mycobacterium abscesses in patients on continuous ambulatory peritoneal dialysis," Saudi Journal of Kidney Diseases and Transplantation, vol. 23, no. 2, pp. 321-324, 2012.

[85] H. I. Hakami, A. A. Alhazmi, and A. A. Alrajhi, "Mycobacterium abscessus peritonitis associated with laparoscopic gastric banding," BMC Infectious Diseases, vol. 13, no. 1, article 323, 2013.

[86] H. Almoallim, L. Alharbi, Z. Alshareef, and G. Wali, "A case of erosive polyarthritis in a patient diagnosed with a suspicion of atypical mycobacteria," Open Rheumatology Journal, vol. 7, no. 1, pp. 64-66, 2013.

[87] A. M. Somily, A. R. Al-Anazi, H. A. Babay et al., "Mycobacterium chelonae complex bacteremia from a post-renal transplant patient: case report and literature review," Japanese Journal of Infectious Diseases, vol. 63, no. 1, pp. 61-64, 2010.

[88] S. Wali, "Mycobacterium chelonae empyema with bronchopleural fistula in an immunocompetent patient," Annals of Thoracic Medicine, vol. 4, no. 4, pp. 213-215, 2009.

[89] A. F. Al-Mobeireek, "Rapidly growing mycobacterial pulmonary infection in association with severe gastroesophageal reflux disease," Saudi Medical Journal, vol. 23, no. 7, pp. 854856, 2002.

[90] R. AlKhodair and S. Al-Khenaizan, "Fish tank granuloma: misdiagnosed as cutaneous leishmaniasis," International Journal of Dermatology, vol. 49, no. 1, pp. 53-55, 2010.

[91] A. M. Hakawi and A. A. Alrajhi, "Septic arthritis due to Mycobacterium szulgai in a patient with human immunodeficiency virus: case report," Scandinavian Journal of Infectious Diseases, vol. 37, no. 3, pp. 235-236, 2005.

[92] H. Al Soub, E. Al-Maslamani, and M. Al-Maslamani, "Mycobacterium fortuitum abdominal wall abscesses following liposuction," Indian Journal of Plastic Surgery, vol. 41, no. 1, pp. 58-61, 2008.

[93] H. Al Soub, M. Al Maslamani, J. Al Khuwaiter, Y. El Deeb, and M. Abu Khattab, "Myocardial abscess and bacteremia complicating mycobacterium fortuitum pacemaker infection: case report and review of the literature," The Pediatric Infectious Disease Journal, vol. 28, no. 11, pp. 1032-1034, 2009.

[94] K. Al-Benwan, S. Ahmad, E. Mokaddas, M. Johny, and M. Kapoor, "Diagnosis of endocarditis caused by Mycobacterium abscessus," Annals of Saudi Medicine, vol. 30, no. 5, pp. 408-411, 2010 . 
[95] T. K. Marras, P. Chedore, A. M. Ying, and F. Jamieson, "Isolation prevalence of pulmonary non-tuberculous mycobacteria in Ontario, 1997-2003," Thorax, vol. 62, no. 8, pp. 661-666, 2007.

[96] P. C. A. M. Buijtels, M. A. B. van der Sande, S. Parkinson, H. A. Verbrugh, P. L. C. Petit, and D. van Soolingen, "Isolation of nontuberculous mycobacteria at three rural settings in Zambia; A Pilot Study," Clinical Microbiology and Infection, vol. 16, no. 8, pp. 1142-1148, 2010. 


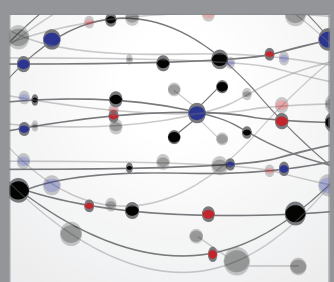

The Scientific World Journal
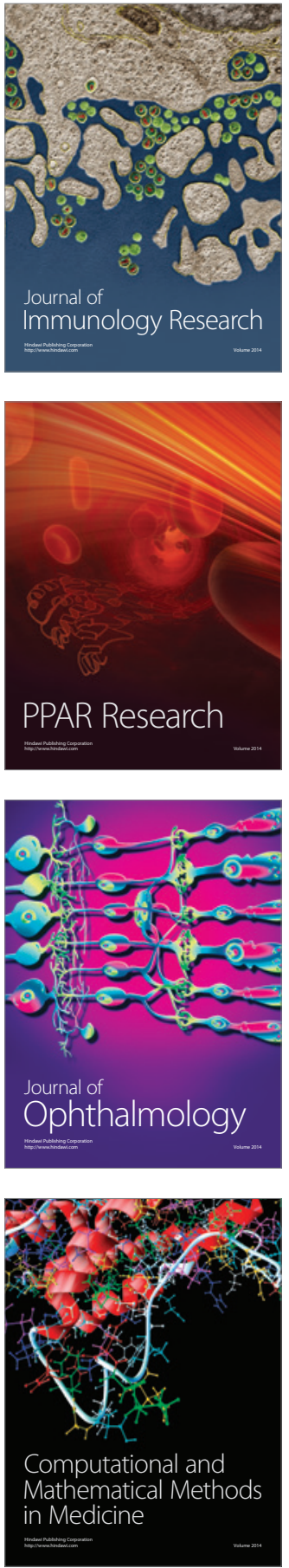

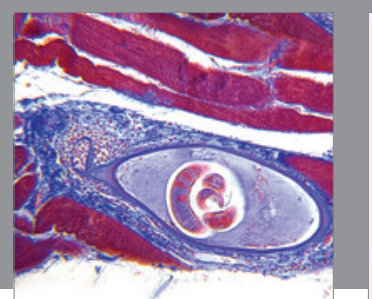

Gastroenterology Research and Practice
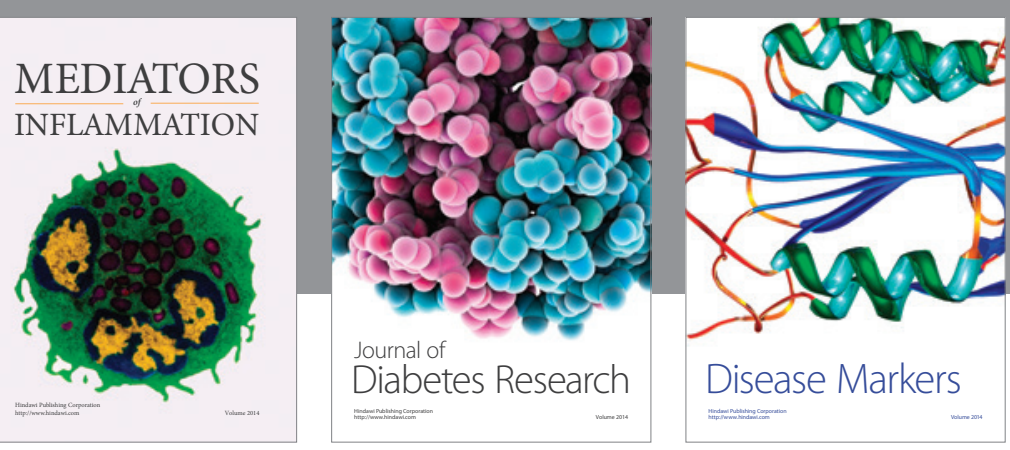

Disease Markers

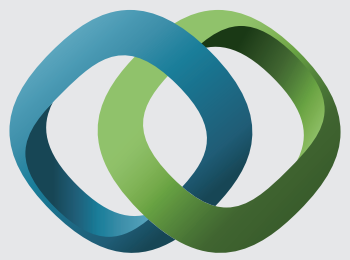

\section{Hindawi}

Submit your manuscripts at

https://www.hindawi.com
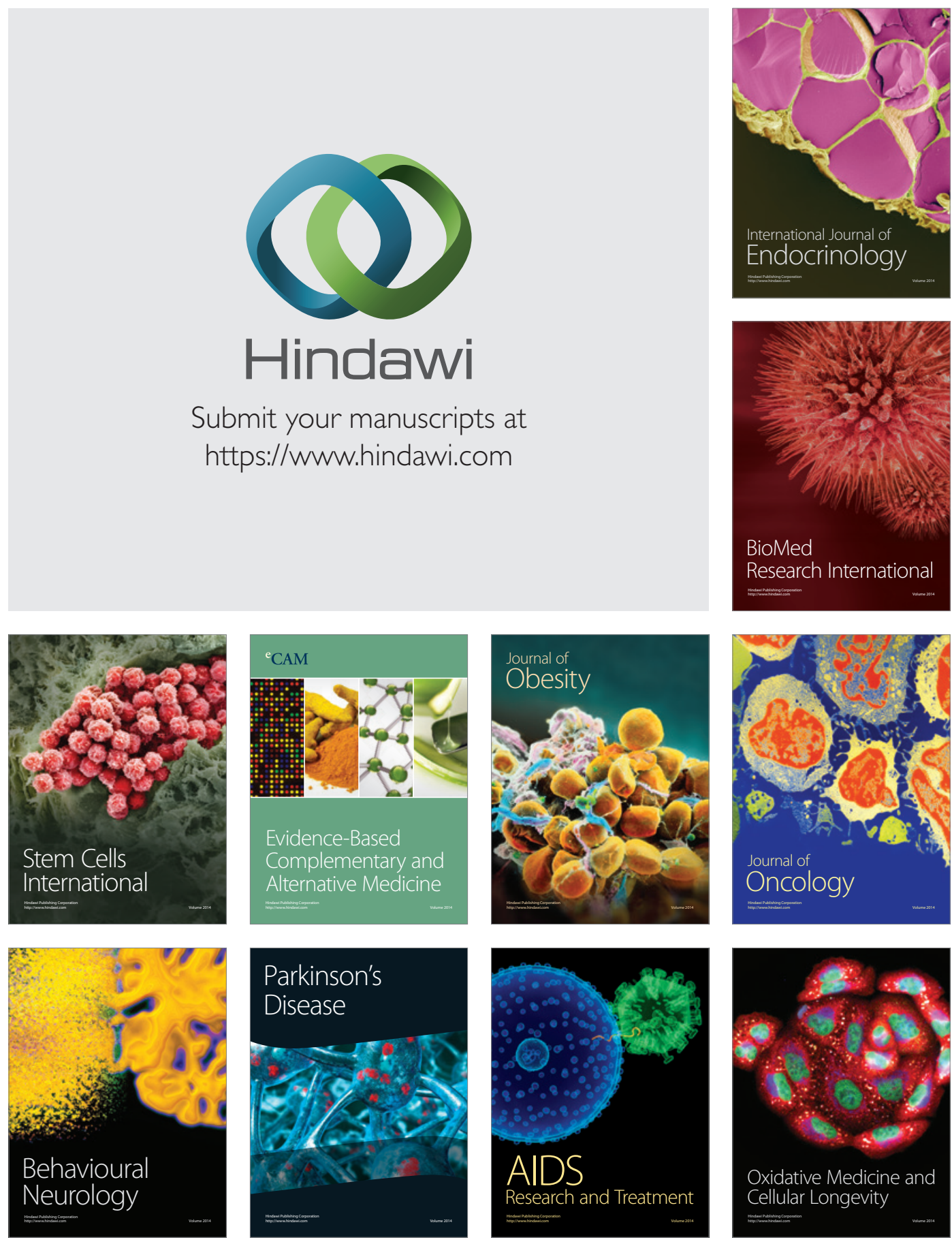